\title{
UNIQUE FACTORISATION OF NORMAL ELEMENTS IN NON-COMMUTATIVE RINGS
}

\author{
by D. A. JORDAN \\ (Received 22 October, 1987; revised 18 January, 1988)
}

1. Introduction. In the literature there are several generalisations to noncommutative rings of the notion of a unique factorisation domain from commutative algebra. This paper follows in the spirit of $[1,3]$ and is set in the context of Noetherian rings. In [3], A. W. Chatters and the author defined a Noetherian UFR (unique factorisation ring) to be a prime Noetherian ring $R$ in which every non-zero prime ideal contains a prime ideal generated by a non-zero normal element $p$, that is, by an element $p$ such that $p R=R p$. The class of Noetherian UFRs includes the Noetherian UFDs studied by Chatters in [1], while a commutative Noetherian ring is a UFR if and only if it is a UFD in the usual sense. For a Noetherian UFR $R$, the following are simple consequences of the definition:

(i) every non-zero ideal of $R$ contains a non-zero normal element;

(ii) the set $N(R)$ of non-zero normal elements of $R$ is a unique factorisation monoid in the sense of [4, Chapter 3].

These two properties provide some justification for the terminology of [3], in that they establish uniqueness of factorisation of a set of elements which is, loosely speaking, dense relative to the ideal structure of the ring.

It is the purpose of this paper to study the class of those prime Noetherian rings, to be termed Noetherian UFN-rings, satisfying (i) and (ii) above. Some examples of Noetherian UFN-rings which are not UFRs will be exhibited in Section 3. In Section 4 we shall identify a chain of five distinct classes of Noetherian UFN-rings, with the class of Noetherian UFRs at the bottom and the full class of Noetherian UFN-rings at the top. Motivation for the defining conditions of these classes comes variously from the examples in Section 3, from considerations of straightforward generalisations of alternative characterisations of commutative UFDs and from consideration of the correspondence between the set of height one prime ideals of the ring and the set of irreducible normal elements of the ring.

The fact that any commutative Noetherian UFD is integrally closed was generalised in [3, Theorem 2.4] which stated that any Noetherian UFR is a maximal order. In Section 5 we shall see that this result does not generalise to Noetherian UFN-rings. Indeed for a ring $R$ in the second smallest of the five classes to be a maximal order it must be a Noetherian UFR.

In [3, Theorem 3.1] it was shown that the class of Noetherian UFRs is closed under polynomial extension. We have been unable to give a complete answer to the corresponding question for the class of Noetherian UFN-rings. We have obtained positive answers for some special cases including those of domains and algebras over infinite fields. Details of these will appear elsewhere. In Section 3 of the present paper we exhibit

Glasgow Math. J. 31 (1989) 103-113. 
an example of a Noetherian UFN-ring $R$ such that $R[x]$ is also a Noetherian UFN-ring but the Laurent polynomial ring $R\left[x, x^{-1}\right]$ is not.

I wish to thank Arthur Chatters and Camilla Jordan for their helpful comments.

2. Definitions, notation and preliminary results. Throughout this paper the term "ring" means "associative ring with an identity element" and the term "Noetherian" means "right and left Noetherian". Let $R$ be a ring. An element $a$ of $R$ is said to be normal if $a R=R a$. The set of all non-zero normal elements of $R$ will be denoted $N(R)$. Suppose now that the ring $R$ is prime. Note that $N(R)$ is a submonoid of the multiplicative monoid of $R$ containing the group $U(R)$ of units of $R$ and that all elements of $N(R)$ are regular in $R$. In the notation of $[4, \mathrm{p} .156], N(R)$ coincides with $I(R)$, the set of invariant elements, and is an invariant cancellation monoid. Following [4], we say that $N(R)$ is a $U F$-monoid if the factor monoid $N(R) / U(R)$ is free commutative.

Let $a \in N(R)$ and $r \in R$. We shall say that $a$ divides $r$, or that $r$ is divisible by $a$, if there exists $s \in R$ such that $a s=r$. Since $a$ is normal, this is equivalent to the existence of $t \in R$ such that $t a=r$; so there is no need to distinguish between right and left divisibility. If $r$ is also normal and $a s=r$ then it is easy to check that $s$ is normal; so divisibility of $r$ by $a$ in the ring $R$ is equivalent to divisibility of $r$ by $a$ in the monoid $N(R)$. Whenever we use the term greatest common divisor of finitely many elements of $N(R)$, this should be interpreted in the context of the monoid $N(R)$ rather than of the ring $R$. The term "associates" will only be used when referring to normal elements and there is then no need to distinguish between right and left associates.

For $a, b \in N(R), a R \subseteq b R$ if and only if $a N(R) \subseteq b N(R)$; so if $R$ is Noetherian then $N(R)$ satisfies the ascending chain condition on principal ideals.

Let $p \in N(R)$. We shall say that $p$ is prime in $N(R)$ if, for all $a, b \in N(R), p$ divides $a b$ implies $p$ divides $a$ or $p$ divides $b$. If $p R$ is a prime ideal of $R$, we shall say that $p$ is prime in $R$. Clearly if $p$ is prime in $R$ then it is prime in $N(R)$ but the converse is false, as can be seen from the examples in Section 3. We shall say that $p$ is an irreducible element of $N(R)$, or is irreducible in $N(R)$, if it cannot be written as the product of two elements of $N(R) \backslash U(R)$.

The following proposition is a special case of [4, Chapter 3, Theorem 1.1 and Corollary 1.2].

Proposition 2.1. For a prime Noetherian ring $R$, the following are equivalent:

(i) $N(R)$ is a UF-monoid;

(ii) any two elements of $N(R)$ have a greatest common divisor;

(iii) for all a, $b \in N(R)$, there exists $c \in N(R)$ such that $a N(R) \cap b N(R)=c N(R)$;

(iv) every irreducible element of $N(R)$ is prime in $N(R)$.

As in [3], we say that a ring $R$ is a Noetherian UFR if it is a Noetherian prime ring in which every non-zero prime ideal of $R$ contains an element which is prime in $R$. We shall say that a ring $R$ is conformal if every non-zero ideal of $R$ contains a non-zero normal element of $R$. If $R$ is a prime Noetherian conformal ring such that $N(R)$ is a UF-monoid, we shall say that $R$ is a Noetherian UFN-ring. 
Proposition 2.2. Let $R$ be a prime Noetherian ring. Then $R$ is a Noetherian UFR if and only if $R$ is conformal and every irreducible element of $N(R)$ is prime in $R$.

Proof. Let $R$ be a Noetherian UFR. Since every non-zero prime ideal of $R$ intersects $N(R)$ non-trivially, so does every non-zero ideal of $R$. Let $p$ be an irreducible element of $N(R)$ and $P$ a prime ideal of $R$ minimal over $p R$. By Jategaonkar's principal ideal theorem [2, Corollary 3.5] $P$ has height one and so $P=a R$ for some $a \in N(R)$. Since $p$ is irreducible in $N(R)$, it follows that $p R=a R=P$ and so $p$ is prime in $R$.

Conversely, suppose that $R$ is conformal and that every irreducible element of $N(R)$ is prime in $R$. Let $P$ be a non-zero prime ideal of $R$ and choose $a \in P \cap N(R)$. Since $R$ is prime Noetherian, $a$ can be written as a product of irreducible elements of $N(R)$ and one of these, $p$ say, must belong to $P$. Thus $P$ contains the prime ideal $p R=R p$ and $R$ is a Noetherian UFR.

A commutative Noetherian domain is a UFD if and only if the intersection of any two principal ideals is principal. This characterisation has proved useful in the commutative theory, for example see $[12, \mathrm{p} .55,90]$, and can be generalised to the non-commutative case in two obvious ways, namely to (iii) of Proposition 2.1 or to the corresponding statement with " $a N(R) \cap b N(R)=c N(R)$ " replaced by " $a R \cap b R=c R$ ". We next show that the latter statement is true if and only if the irreducible elements of $N(R)$ have a property intermediate between being prime in $R$ and being prime in $N(R)$.

Proposition 2.3. For a prime Noetherian ring $R$, the following are equivalent:

(i) for all $a, b \in N(R)$, there exists $c \in N(R)$ such that $a R \cap b R=c R$;

(ii) if $p$ is an irreducible element of $N(R)$ and $p$ divides $a b$, where $a \in N(R)$ and $b \in R$, then $p$ divides $a$ or $p$ divides $b$;

(iii) if $p$ is an irreducible element of $N(R)$ and $p$ divides $a b$, where $a \in R$ and $b \in N(R)$, then $p$ divides $a$ or $p$ divides $b$.

Proof. Suppose that (i) holds and let $a, b \in N(R)$. Then there exists $c \in N(R)$ such that $a R \cap b R=c R$ and it is easy to check that $a N(R) \cap b N(R)=c N(R)$. By Proposition 2.1 , it follows that irreducible normal elements of $N(R)$ are prime in $N(R)$. Let $p$ be an irreducible element of $N(R)$ and let $a \in N(R), b, t \in R$ be such that $p t=a b$. Suppose that $p$ does not divide $a$. By (i), there exists $c \in N(R)$ such that $a R \cap p R=c R$. There exist $u, v \in R$ such that $c=a u=p v$ and, since $c, a, p$ are normal, $u$ and $v$ are normal. Since $p$ is prime in $N(R)$ and does not divide $a, p$ divides $u$. But $p t=a b \in p R \cap a R=c R=a u R$; so, by the regularity of $a, b \in u R$. Since $p$ divides $u$, it must also divide $b$. Thus (i) implies (ii).

Suppose now that (ii) holds. Then irreducible elements of $N(R)$ are prime in $N(R)$ and so, by Proposition $2.1, N(R) / U(R)$ is free commutative and any two elements of $N(R)$ have a greatest common divisor in $N(R)$. In showing that (i) holds there is no loss of generality in assuming that $a, b$ have greatest common divisor 1 and that $a \notin U(R)$. If $a$ is an irreducible element of $N(R)$ then $a$ does not divide $b$ and, by (ii), $a R \cap b R=b a R$. If $a$ is not irreducible in $N(R)$ then we may write $a=p a_{1}$, where $a_{1} \in N(R)$ and $p$ is an irreducible element of $N(R)$ not dividing $b$, and, by induction, we may assume that there exists $c_{1} \in N(R)$ such that $a_{1} R \cap b R=c_{1} R$. Let $x=a r=b s \in a R \cap b R$. Then $p$ divides $b s$; 
so, by (ii), $s=p s_{1}$ for some $s_{1} \in R$ and, since $N(R) / U(R)$ is commutative, $p a_{1} r=a r=$ $b s=b p s_{1}=p b u s_{1}$ for some $u \in U(R)$. By the regularity of $p, a_{1} r=b u s_{1} \in a_{1} R \cap b R$. Thus $a_{1} r \in c_{1} R$ and so $x=p a_{1} r \in p c_{1} R$. Thus $a R \cap b R \subseteq p c_{1} R$. The reverse inclusion is clear.

This completes the proof that (i) is equivalent to (ii). The equivalence of (i) and (iii) follows by the symmetry of (i) which can be seen by rewriting $a R, b R$ and $c R$ in (i) as $R a, R b$ and $R c$.

Let $a$ be a regular normal element of a ring $R$. There is an induced automorphism $\alpha_{a}$ of $R$ such that $a r=\alpha_{a}(r) a$ for all $r \in R$. For any automorphism $\alpha$ of $R$, an ideal $I$ of $R$ is said to be an $\alpha$-ideal of $R$ if $\alpha(I) \subseteq I$, and to be $\alpha$-prime if it is an $\alpha$-ideal of $R$ such that, for all $\alpha$-ideals $A, B$ of $R, A B \subseteq I$ implies $A \subseteq I$ or $B \subseteq I$. We shall require the following results from [6].

LEMMA 2.4. Let $R$ be a Noetherian ring and $\alpha$ an automorphism of $R$.

(i) An ideal $I$ of $R$ is an $\alpha$-ideal if and only if $I=\alpha(I)$.

(ii) Any $\alpha$-prime ideal of $R$ is semiprime.

3. Examples. In this section we shall discuss some examples of Noetherian UFN-rings which are not UFRs. We begin with two classes of examples constructed from a commutative Noetherian UFD $R$ and a prime element $p$ of $R$. For such $R$ and $p$, we let $\Gamma(R, p)$ denote the subring of the $2 \times 2$ matrix ring $M_{2}(R)$ generated by the matrices

$$
\left[\begin{array}{ll}
1 & 0 \\
0 & 1
\end{array}\right],\left[\begin{array}{ll}
1 & 0 \\
0 & 0
\end{array}\right],\left[\begin{array}{ll}
0 & p \\
0 & 0
\end{array}\right] \text { and }\left[\begin{array}{ll}
0 & 0 \\
1 & 0
\end{array}\right] \text {. }
$$

Thus $\Gamma(R, p)=\left[\begin{array}{cc}R & p R \\ R & R\end{array}\right]$.

We let $\Delta(R, p)$ denote the subring of $M_{2}(R)$ generated by the matrices

$$
\left[\begin{array}{ll}
1 & 0 \\
0 & 1
\end{array}\right],\left[\begin{array}{ll}
p & 0 \\
0 & 0
\end{array}\right],\left[\begin{array}{ll}
0 & p \\
0 & 0
\end{array}\right] \text { and }\left[\begin{array}{ll}
0 & 0 \\
1 & 0
\end{array}\right] \text {. }
$$

Thus a typical element of $\Delta(R, p)$ has the form $\left[\begin{array}{cc}a & p b \\ c & a+p d\end{array}\right]$, where $a, b, c, d \in R$. An example of a ring of the form $\Delta(R, p)$ is discussed in [2, p. 157].

Proposition 3.1. Let $R$ be a commutative Noetherian UFD and $p$ be a prime element of $R$. Then the rings $\Gamma(R, p)$ and $\Delta(R, p)$ are Noetherian prime conformal rings. Neither $\Gamma(R, p)$ nor $\Delta(R, p)$ is a Noetherian UFR.

Proof. In both cases the generators for the ring given above are generators for the ring as a module over its Noetherian centre. Hence the ring is Noetherian. It is easy to check, using the presence in the ring of $p e_{11}, p e_{12}, e_{21}$ and $p e_{22}$ (where the elements $e_{i j}$ are the usual matrix units), that each ring is prime. That each ring is conformal follows from the fact, easily established by direct computation involving adjoints or by quoting standard results of PI-theory, that every non-zero ideal of the ring intersects the centre non-trivially. 
Let $A$ denote the matrix $\left[\begin{array}{ll}0 & p \\ 1 & 0\end{array}\right]$. Then $A$ is a normal element of both $\Gamma(R, p)$ and $\Delta(R, p)$ because both rings are invariant under the inner automorphism of $M_{2}(Q)$ induced by $A$, where $Q$ denotes the quotient field of $R$. This automorphism maps a typical element $\left[\begin{array}{cc}a & p b \\ c & d\end{array}\right]$ of $\Gamma(R, p)$ to $\left[\begin{array}{cc}d & p c \\ b & a\end{array}\right]$.

Let $P_{1}=\left[\begin{array}{cc}p R & p R \\ R & R\end{array}\right]$ and $P_{2}=\left[\begin{array}{cc}R & p R \\ R & p R\end{array}\right]$. These are ideals of $\Gamma(R, p)$ with factor rings isomorphic to $R$ and so are prime. Note that $A \Gamma(R, p)=P_{1} \cap P_{2}$; so that $A$ is not prime in $\Gamma(R, p)$. Any non-trivial factorisation of $A$ in $N(\Gamma(R, p))$ would give a non-trivial factorisation of det $A=-p$ in $R$, for if $B \in \Gamma(R, p)$ is a unit in $M_{2}(R)$ then $B^{-1} \in \Gamma(R, p)$. Thus $A$ is an irreducible element of $N(\Gamma(R, p))$ which is not prime in $\Gamma(R, p)$. By Proposition $2.2, \Gamma(R, p)$ is not a Noetherian UFR.

Now consider the ring $\Delta(R, p)$ and let $P=\left[\begin{array}{cc}p R & p R \\ R & p R\end{array}\right]$, a prime ideal of $\Delta(R, p)$ with factor ring isomorphic to $R$. Then $P^{2} \subset A \Delta(R, p) \subset P$, both inclusions being strict. In fact $P^{2}=A P$. Thus $A$ is not prime in $\Delta(R, p)$ but, as for $\Gamma(R, p)$ above, it is an irreducible element of $N(\Delta(R, p))$. Thus $\Delta(R, p)$ is not a Noetherian UFR.

Proposition 3.2. Let $R$ be a commutative Noetherian UFD and $p$ a prime element of $R$. Then the ring $\Gamma(R, p)$ is a Noetherian UFN-ring.

Proof. Let $A$ be as in the proof of Proposition 3.1. We shall show that, up to associates, the irreducible elements of $N(\Gamma(R, p))$ are $A$ and the irreducible elements of the centre of $\Gamma(R, p)$ (which we identify with $R$ ) apart from $p$, which factorises as $A^{2}$. Since $N(R) / U(R)$ is free commutative, so is $N(\Gamma(R, p)) / U(\Gamma(R, p))$. By Proposition 3.1, it will follow that $\Gamma(R, p)$ is a Noetherian UFN-ring.

Let $B=\left[\begin{array}{cc}i & p j \\ k & l\end{array}\right] \in \Gamma(R, p)$ be a normal non-unit with $i, j, k, l$ coprime in $R$ and let $\delta=\operatorname{det} B$, which is necessarily non-zero. The ring $\Gamma(R, p)$ is invariant under the mapping $C \mapsto B^{-1} C B$ and calculation of $B^{-1} p e_{12} B$ and $B^{-1} e_{21} B$ reveals that $\delta^{-1} i^{2}, \delta^{-1} p j^{2}, \delta^{-1} p k^{2}$ and $\delta^{-1} l^{2} \in R$. It follows that $p$ and its associates are the only possible prime factors of $\delta$ and that $p^{2}$ does not divide $\delta$. Since $B$ is a non-unit in $\Gamma(R, p), \delta$ is a non-unit of $R$. Thus $\delta$ is an associate of $p$ and it follows that $i \in p R$ and $l \in p R, i=p m, l=p n$, say. But then $B=\left[\begin{array}{cc}j & p m \\ n & k\end{array}\right]\left[\begin{array}{ll}0 & p \\ 1 & 0\end{array}\right]=C A$, say. Since $j k-p m n=-\delta p^{-1}$ is a unit in $R, C$ is a unit in $M_{2}(R)$ and hence in $\Gamma(R, p)$. Thus $B$ is an associate of $A$. It follows from these calculations that the irreducible elements of $N(\Gamma(R, p))$ are as claimed above. Thus $\Gamma(R, p)$ is a Noetherian UFN-ring.

By contrast with $\Gamma(R, p)$, it turns out that $\Delta(R, p)$ is rarely a Noetherian UFN-ring. Proposition 3.3. Let $R$ be a commutative Noetherian UFD and let $p$ be a prime 
element of $R$. The ring $\Delta(R, p)$ is a Noetherian UFN-ring if and only if, for all elements $j, k$ of $R$ and units $u$ of $R, j k \equiv u \bmod p R$ implies $j \equiv k \bmod p R$.

Proof. Suppose that there exist $j, k, u \in R$, with $u$ a unit, such that $j k \equiv u \bmod p R$ but $j \neq k \bmod p R$. Let $D$ denote the matrix $\left[\begin{array}{ll}j & p \\ r & k\end{array}\right]$, where $j k-u=p r$. Note that $D$ is a unit in $M_{2}(R)$ and that neither $D$ nor $D^{-1}$ belongs to $\Delta(R, p)$. However it can be calculated that $D \Delta(R, p) D^{-1}=\Delta(R, p)=D^{-1} \Delta(R, p) D$,

$$
A D=\left[\begin{array}{cc}
p r & p k \\
j & p
\end{array}\right] \in \Delta(R, p) \text { and } D^{-1} A=\left[\begin{array}{cc}
-p u^{-1} & p k u^{-1} \\
j u^{-1} & -p r u^{-1}
\end{array}\right] \in \Delta(R, p)
$$

Consequently $A D$ and $D^{-1} A$ are normal elements of $\Delta(R, p)$. But $(A D)\left(D^{-1} A\right)=A^{2}=p$ and, since $D, D^{-1} \notin \Delta(R, p), A$ divides neither $A D$ nor $D^{-1} A$ in $\Delta(R, p)$. So $A$ is not prime in $N(\Delta(R, p))$ and, by Proposition 2.1 , the prime Noetherian ring $\Delta(R, p)$ is not a Noetherian UFN-ring.

Conversely suppose that, for all elements $j, k$ of $R$ and units $u$ of $R, j k \equiv u \bmod p R$ implies $j \equiv k \bmod p R$. Follow the proof of Proposition 3.2, with $\Delta(R, p)$ replacing $\Gamma(R, p)$, up to the point where $B$ factorises as $C A$, with $C=\left[\begin{array}{cc}j & p m \\ n & k\end{array}\right]$, and $j k-p m n$ is a unit in $R$. By supposition, $C \in \Delta(R, p)$ and so is a unit in $\Delta(R, p)$. As in the proof of Proposition 3.2, $B$ is an associate of $A$ and $\Delta(R, p)$ is a Noetherian UFN-ring.

REMARK 3.4. Examples in which $\Delta(R, p)$ is a Noetherian UFN-ring include the following: (i) $p=2$ and $R=\mathbb{Z}$; (ii) $p=2$ and $R$ is the localisation of $\mathbb{Z}$ at the maximal ideal $2 \mathbb{Z}$; (iii) $p=2$ and $R$ is a polynomial ring $\mathbb{Z}\left[x_{1}, x_{2}, \ldots, x_{n}\right]$ over $\mathbb{Z}$ in finitely many commuting indeterminates; (iv) $p=1+i$ and $R$ is the ring of Gaussian integers; (v) $p=x_{i}$ and $R$ is a polynomial ring $\mathbb{Z}_{2}\left[x_{1}, x_{2}, \ldots, x_{n}\right]$ over the field $\mathbb{Z}_{2}$ of two elements in finitely many commuting indeterminates.

Examples in which $\Delta(R, p)$ is not a Noetherian UFN-ring include those where $R=\mathbb{Z}$ and $p$ is an odd prime, those where $R$ is a polynomial ring over a field of order greater than 2 in finitely many commuting indeterminates and $p$ is one of the indeterminates, and those where $R=S\left[x, x^{-1}\right]$ is the Laurent polynomial ring over any commutative Noetherian UFD $S$ and $p$ is any prime element of $S$. To see that such a ring $S\left[x, x^{-1}\right]$ fails to satisfy the condition in Proposition 3.3, take $j=u=x$ and $k=1$.

These examples reveal some surprising bad behaviour of the class of Noetherian UFN-rings. Note that $\Delta(\mathbb{Z}, 2)$ and $\Delta(\mathbb{Z}, 2)[x]$, which is isomorphic to $\Delta(\mathbb{Z}[x], 2)$, are Noetherian UFN-rings, but that $\Delta(\mathbb{Z}, 2)\left[x, x^{-1}\right]$, which is isomorphic to $\Delta\left(\mathbb{Z}\left[x, x^{-1}\right], 2\right)$, is not. Thus the class of Noetherian UFN-rings is closed under neither Laurent polynomial extensions nor localisation at the set of powers of a central element.

Remark 3.5. The proof of Proposition 3.1 reveals some significant difference in the behaviour of the irreducible normal elements in the rings $\Gamma(R, p)$ and $\Delta(R, p)$, even in cases where the latter is a Noetherian UFN-ring. In $\Gamma(R, p)$ the ideal generated by $A$ is semiprime, being equal to $P_{1} \cap P_{2}$, see the proof of Proposition 3.1. Note that the 
automorphism $\alpha_{A}$ transposes $P_{1}$ and $P_{2}$; so that $A \Gamma(R, p)$ is $\alpha_{A}$-prime. However in $\Delta(R, p)$ we have $P^{2}=A P \subset A \Delta(R, p) \subset P$ and $\alpha_{A}(P)=P$; so that $A \Delta(R, p)$ is neither $\alpha_{A}$-prime nor semiprime. In both rings every irreducible normal element apart from $A$ generates a prime ideal.

The next example serves to illustrate that a Noetherian UFN-ring need not satisfy the equivalent conditions of Proposition 2.3.

EXAmple 3.6. Let $K$ be a field, $A$ the polynomial ring $K[x, y]$ over $K$ in two commuting indeterminates and $M$ the maximal ideal $x A+y A$. Let $R$ be the subring $\left[\begin{array}{ll}A & M \\ A & A\end{array}\right]$ of $M_{2}(A)$. Routine arguments and calculations, similar to those given in the proofs of 3.1 and 3.2, show that $R$ is prime Noetherian and conformal and that the normal elements of $R$ are, up to associates in $R$, the elements of its centre, which we identify with $A$. Consequently $R$ is Noetherian UFN-ring.

Note that $x$ and $y$ are irreducible elements of $N(R)$ and that, although the matrix unit $e_{12} \notin R, x e_{12} \in R$ and $y e_{12} \in R$. Since $x\left(y e_{12}\right)=y\left(x e_{12}\right)$ and $x$ divides neither $y$ nor $x e_{12}, R$ does not satisfy the equivalent statements of Proposition 2.3. The ideal $x R \cap y R$ is not generated by a normal element of $R$.

In this example there is a bijection between the set of associate classes of irreducible elements of $N(R)$ and the set of height one prime ideals of $R$ given by

$$
p \leftrightarrow P=\left[\begin{array}{cc}
p A & p A \cap M \\
p A & p A
\end{array}\right] .
$$

Note that if $p \notin M$ then $P=p R$ and that if $p \in M$ then $P \neq p R$ but $P^{2}=p P$.

In all the examples of Noetherian rings considered so far each height one prime ideal contains, up to associates, a unique irreducible element of $N(R)$. The next example shows that this is not always the case.

Example 3.7. Let $K$ be a field of characteristic zero and let $A$ be the factor ring $K[u, v, w, t: u t=v w]=K[U, V, W, T] /(U T-V W)$ of the commutative polynomial ring $K[U, V, W, T]$. Let $\gamma$ be the $K$-derivation

$$
U \partial / \partial U+V \partial / \partial V+(U+W) \partial / \partial W+(V+T) \partial / \partial T
$$

of $K[U, V, W, T]$. Note that $\gamma(U T-V W)=2(U T-V W)$; so that $\gamma$ induces a $K$ derivation $\delta$ of $A$ such that $\delta(u)=u, \delta(v)=v, \delta(w)=w+u$ and $\delta(t)=v+t$. Let $R$ be the formal ring of differential operators or Ore extension $A[x ; \delta]$. Thus $R$ is a Noetherian domain, see for example [4, p. 53]. We shall show that

(i) $N(R)$ is the set of non-zero homogeneous polynomials in $u$ and $v$ with coefficients in $K$. Hence $N(R)$ is a UF-monoid;

(ii) $R$ is conformal; $N(R)$.

(iii) $R$ has a height one prime ideal $P$ which contains all the irreducible elements of

The ring $A$ has as subrings three polynomial rings $A_{1}=K[u, v, t], A_{2}=K[u, v]$ and $A_{3}=K[u]$. Note that each of these is invariant under $\delta$. Let $H$ denote the set of non-zero 
homogeneous elements of $A_{2}$ and observe that if $f \in H$ has degree $d$ then $\delta(f)=d f$ and hence that $f \in N(R)$. Thus $H \subseteq N(R)$. To establish the reverse inclusion, let $a \in R$ be normal of degree $n$ in $x, a=a_{n} x^{n}+\ldots+a_{0}$. If $n>0$ then $a u-u a=n a_{n} \delta(u) x^{n-1}+$ terms of lower degree $=n a_{n} u x^{n-1}+$ terms of lower degree. But this element must belong to the ideal $a R$, which is not possible since $A$ is a domain and $a u-u a$ is non-zero with degree less than that of $a$. Thus $n=0$ and $a \in A$. Since $\delta(a)=x a-a x \in a R \cap A=a A$ there must exist $b \in A$ such that $\delta(a)=b a$. Straightforward computations in the graded rings $A, A_{1}$ and $A_{2}$ can now be used to show successively that $b \in K, a \in A_{1}, a \in A_{2}$ and finally that $a \in H$. Thus $N(R)=H$ which is clearly a UF-monoid.

To show that $R$ is conformal, it is enough to show that if $P$ is a non-zero prime ideal of $R$ then $P \cap N(R)$ is non-empty. By [8, Lemma 1] and [7, Lemmas 1.3 and 2.1 and Theorem 2.2], $P \cap A$ is a non-zero prime ideal of $A$ and is invariant under $\delta$. So $P \cap A=P^{\prime} /(U T-V W)$ for some prime ideal $P^{\prime}$ of height at least 2 in $K[U, V, W, T]$. It follows, using [10, Theorem 37], that for some $i, 1 \leqq i \leqq 3, P \cap A_{i}$ is a height one prime ideal of $A_{i}$. By [10, Theorem 5], $P \cap A_{i}=a A_{i}$ for some non-zero $a \in A_{i}$. But $\delta\left(A_{i}\right) \subseteq A_{i}$ and $\delta(P) \subseteq P$; so $\delta(a) \in a A_{i}$. It follows that $a \in N(R)$ and hence that $R$ is conformal.

Now let $Q$ be the prime $\delta$-invariant ideal $u A+v A$ of $A$ and let $P=Q R=u R+v R$ which, by [7, Lemma 1.3], is a prime ideal of $R$. Any prime ideal of $A$ containing $u$ must contain either $v$ or $w$; so $Q$ is minimal over $w A$ and, by the principal ideal theorem, has height one. It follows, by [8, Lemma 1] and [7, Lemmas 1.3 and 2.1 and Theorem 2.2], that $P$ has height one in $R$. Clearly $N(R)=H \subseteq P$. Thus (iii) holds.

Nevertheless, in the ring $R$, there is a strong correspondence between the height one prime ideals and the irreducible elements of $N(R)$. This is simplest to describe in the case where the field $K$ is algebraically closed. In this case the irreducible elements of $N(R)$ are the homogeneous polynomials in $u$ and $v$ of degree 1 . Thus

$$
N(R)=\{\lambda u+\mu v: \lambda, \mu \in K, \text { not both zero }\} .
$$

It can be checked that, for $a=\lambda u+\mu v \in N(R), a R=P \cap P_{\lambda, \mu}$, where $P=u R+v R$ as above and $P_{\lambda, \mu}=(\lambda u+\mu v) R+(\lambda w+\mu t) R$ is another height one prime ideal of $R$. The mapping $Q \mapsto P \cap Q$ is a bijection from the set of height one primes of $R$ different from $P$ to the set of ideals of $R$ generated by irreducible elements of $N(R)$.

\section{Five classes of UFN-rings.}

TheOREM 4.1. Let $R$ be a prime Noetherian ring and consider the following statements:

(1) $R$ is a Noetherian UFR;

(2) $R$ is conformal and, for every irreducible element a of $N(R)$, aR is an $\alpha_{a}$-prime ideal of $R$;

(3) $R$ is conformal and, for all $a, b \in N(R)$, there exists $c \in N(R)$ such that $a R \cap b R=c R$;

(4) $R$ is conformal and each height one prime ideal of $R$ contains, up to associates, $a$ unique irreducible element of $N(R)$;

(5) $R$ is a Noetherian UFN-ring. 
Then (1) implies (2), (2) implies (3), (3) implies (4) and (4) implies (5) but none of the reverse implications are true.

Proof. (1) implies (2). This is immediate from Proposition 2.2.

(2) implies (3). Let $p \in N(R)$ be such that $p R$ is $\alpha_{p}$-prime and let $a \in N(R), b \in R$ such that $p$ divides $a b$. We shall show that $p$ divides $a$ or $p$ divides $b$. By Proposition 2.3, this will establish that (2) implies (3). By [2, Lemma 3.3], $p R$ has the right Artin-Rees property; so there exists a positive integer $n$ such that $a R \cap p^{n} R \subseteq a p R$. Hence $p^{n} a=a p r$ for some $r \in R$. Since $a$ is regular, $\alpha_{a}^{-1}(p)^{n}=p r$. By Lemma 2.4(ii), $p R$ is semiprime; so, since $\alpha_{a}^{-1}(p)$ is normal, $\alpha_{a}^{-1}(p) \in p R$. It follows, by Lemma 2.4(i), that $p R=\alpha_{a}(p R)$. Hence there exists $s \in R$ such that $p a=a s p$. But then $\alpha_{p}(a)=a s \in a R$ and $a R$ is an $\alpha_{p}$-ideal. Since the ideal $\{r \in R: a r \in p R\}$ is then also an $\alpha_{p}$-ideal, it follows, since $p R$ is $\alpha_{p}$-prime, that $a \in p R$ or $b \in p R$.

(3) implies (4). Suppose that (3) holds and that $P$ is a height one prime ideal of $R$ containing two irreducible elements $p, q$ of $N(R)$ which are not associates. Let $P=P_{1}, P_{2}, \ldots, P_{n}$ be the prime ideals of $R$ minimal over $p R$. Since $R$ is Noetherian, there exists a positive integer $k$ such that $\left(P_{1} \cap P_{2} \cap \ldots \cap P_{n}\right)^{k} \subseteq p R$. If $q \in P_{i}$ for all $i$ then $q^{k} \in p R$, but this is not possible by Proposition 2.3. So we can renumber the ideals $P_{i}$ so that $q \in P_{1}, P_{2}, \ldots, P_{m}, q \notin P_{m+1}, \ldots, P_{n}$, where $1 \leqq m<n$. If $i>m$ then $\alpha_{q}\left(P_{i}\right) q=$ $q P_{i} \subseteq P_{i}$ and so, since $q \notin P_{i}$ and is normal, $\alpha_{q}\left(P_{i}\right) \subseteq P_{i}$. By Lemma 2.4, $\alpha_{q}\left(P_{i}\right)=P_{i}$. Thus $\alpha_{q}\left(P_{m+1} \cap \ldots \cap P_{n}\right)=P_{m+1} \cap \ldots \cap P_{n}$ and so

$$
q^{k}\left(P_{m+1} \cap \ldots \cap P_{n}\right)^{k}=\left(q\left(P_{m+1} \cap \ldots \cap P_{n}\right)\right)^{k} \subseteq p R
$$

By Proposition 2.3, $\left(P_{m+1} \cap \ldots \cap P_{n}\right)^{k} \subseteq p R \subseteq P_{1}$. This is a contradiction. Thus (3) implies (4).

(4) implies (5). Suppose that (4) holds and let $p$ be an irreducible element of $N(R)$ and $a, b \in N(R)$ such that $p$ divides $a b$. By Proposition 2.1, it suffices to show that $p$ divides $a$ or $p$ divides $b$. Let $P$ be a prime ideal of $R$ minimal over $p R$ so that, by Jategaonkar's principal ideal theorem [2, Corollary 3.5], $P$ has height one. Either $a \in P$ or $b \in P$. But, up to associates, $p$ is the only irreducible element of $N(R)$ in $P$ and, since $R$ is Noetherian, every element of $N(R)$ factorises as a product of irreducible elements of $N(R)$. Hence either $p$ divides $a$ or $p$ divides $b$, as required.

The rings $\Gamma(R, p)$ constructed in Section 3 satisfy (2), see Remark 3.5, but not (1), see Proposition 3.1.

Let $R$ be the ring obtained by localising $\mathbb{Z}$ at $2 \mathbb{Z}$ and consider the ring $\Delta(R, 2)$ constructed in Section 3. By Remark 3.5, $\Delta(R, 2)$ does not satisfy (2). But the normal elements of $\Delta(R, 2)$, up to associates, are powers of the element $A=\left[\begin{array}{ll}0 & 2 \\ 1 & 0\end{array}\right]$. Hence $\Delta(R, 2)$, which is prime Noetherian conformal by Proposition 3.1, satisfies (3).

The ring of Example 3.6 satisfies (4) but not (3), while the ring of Example 3.7 satisfies (5) but not (4).

In the remaining section of the paper we shall say that a Noetherian UFN-ring is of class $i, 1 \leqq i \leqq 5$, if it satisfies statement (i) of Theorem 4.1. 
5. Maximal orders. In [3, Theorem 2.4] it was shown that if $R$ is a Noetherian UFR, in other words a Noetherian UFN-ring of class 1 , then $R$ is a maximal order. That this result does not generalise to any of the larger classes of Noetherian UFN-rings introduced in Section 4 is clear from the following result and the existence, observed in Section 4, of a Noetherian UFN-ring of class 2 which is not a Noetherian UFR.

THEOREM 5.1. If $R$ is a Noetherian UFN-ring of class 2 and is a maximal order then $R$ is a Noetherian UFR.

Proof. Suppose that $R$ is a Noetherian UFN-ring of class 2 which is not a Noetherian UFR. Then there exists an irreducible element $p$ of $N(R)$ such that the ideal $p R$ is $\alpha$-prime but not prime, where $\alpha=\alpha_{p}$. Let $P_{1}, P_{2}, \ldots, P_{n}$ be the prime ideals of $R$ minimal over $p R$. By Lemma $2.4, p R$ is semiprime; so $p R=P_{1} \cap P_{2} \cap \ldots \cap P_{n}$. Hence $n>1$. Since $\alpha$ permutes the ideals $P_{i}$ and $p R$ is $\alpha$-prime, the ideals $P_{i}$ must form a single orbit under the action of $\alpha$. Thus we may assume that $\alpha\left(P_{1}\right)=P_{2} \neq P_{1}$. Now $p$ is invertible in the quotient ring of $R$ and $P_{1} p^{-1}\left(P_{2} \cap \ldots \cap P_{n}\right) P_{1} \subseteq P_{1} p^{-1} p R=P_{1}$. On the other hand, $P_{1} p^{-1}\left(P_{2} \cap \ldots \cap P_{n}\right) \notin R$, otherwise, since $P_{1} p^{-1}=p^{-1} P_{2}, P_{2}\left(P_{2} \ldots P_{n}\right) \subseteq p R \subseteq P_{1}$, giving $P_{j} \subseteq P_{1}$ for some $j>1$, which is impossible. It follows by [11, I.3.1] that $R$ is not a maximal order.

The proof of Theorem 5.1 can be generalised, using primary decomposition theorems for reflexive ideals in maximal orders, for example [5, Theorem 2.2], to show that if a prime Noetherian ring $R$ has a height one prime ideal which is not invariant under the automorphism of $R$ induced by some normal element of $R$ then $R$ cannot be a maximal order. For other "bad behaviour" which is guaranteed in this situation, see [9].

If $R$ is either of the rings used to show that, in Theorem 4.1, (3) does not imply (2) and (4) does not imply (3) then $R$ has a prime ideal $P$ and a normal element $p$ such that $P \neq p R$ but $P^{2}=p P$, see Remark 3.5 and Example 3.6. It follows that, in the quotient ring of $R,\left(p^{-1} P\right) P \subseteq P$ but $p^{-1} P \nsubseteq R$ so that, by [11, I.3.1], $R$ is not a maximal order. However the ring of Example 3.7 which is a Noetherian UFN-ring of class 5, but not of class 4 , is a maximal order. It is of the form $A[x ; \delta]$, where $A$ is an integrally closed commutative Noetherian domain, and so is a maximal order by [11, I.5.1 and V.2.5]. Thus Theorem 5.1 does not generalise from class 2 to class 5 .

\section{REFERENCES}

1. A. W. Chatters, Non-commutative unique factorisation domains, Math. Proc. Cambridge Philos. Soc. 95 (1984), 49-54.

2. A. W. Chatters and C. R. Hajarnavis, Rings with chain conditions (Pitman, 1980).

3. A. W. Chatters and D. A. Jordan, Non-commutative unique factorisation rings, J. London Math. Soc. (2) 33 (1986), 22-32.

4. P. M. Cohn, Free rings and their relations, London Math. Soc. Monograph No. 19, 2nd edition (Academic Press, 1985).

5. J. H. Cozzens and F. L. Sandomierski, Reflexive primes, localization and primary decomposition in maximal orders, Proc. Amer. Math. Soc. 58 (1976), 44-50. 
6. A. W. Goldie and G. O. Michler, Ore extensions and polycyclic group rings, J. London Math. Soc. (2) 9 (1974), 337-345.

7. D. A. Jordan, Noetherian Ore extensions and Jacobson rings, J. London Math. Soc. (2) 10 (1975), 281-291.

8. D. A. Jordan, Primitive Ore extensions, Glasgow Math. J. 18 (1977), 93-97.

9. D. A. Jordan, Normal elements and completions of non-commutative Noetherian rings, Bull. London Math. Soc. 19 (1987), 417-424.

10. I. Kaplansky, Commutative rings, revised edition (University of Chicago Press, 1974).

11. G. Maury and J. Raynaud, Ordres maximaux au sens de K. Asano, Lecture Notes in Mathematics 808 (Springer, 1980).

12. P. Samuel, Anneaux factoriels (Sociedade de Matemática de São Paulo, 1963).

Department of Pure Mathematics

UNIVERSITY OF SHEFFIELD

Hicks BUILDING

HOUNSFIELD ROAD

SHEFFIELD S3 7RH 